\title{
Teaching Objectives and Teaching Content Point of View the Concept of Sports Teaching and the Comparison of the Chinese Sports Teaching
}

\author{
Bing Zhang ${ }^{1, *}$, Kunling Qin ${ }^{2}$, Qian Yang ${ }^{1}$ \\ ${ }^{1}$ Institute of Physical Education, Huanggang Normal University, Huangzhou 438000, Hubei, China \\ ${ }^{2}$ Yidu Gaobazhou Middle School, Yidu 443300, Hubei, China \\ tiyuxi@qq.com
}

\begin{abstract}
The study purpose: to understand the differences of physical education and for the two countries in the sports teaching target and content to deepen the reform to provide the reference. Methods: the literature material law, field investigation method, interview method, comparative analysis method. Research results show that the two countries in the aspect of teaching objectives and teaching content exist similarities and differences, this has to do with the historical culture and national conditions, such as the two countries have a certain relation.
\end{abstract}

Keywords: the concept of sports; Sports teaching; Teaching methods; Teaching content

\section{INTRODUCTION}

With outstanding Chinese students constitution and health problems, basic education "sports and health course" reform thorough, the 2011 edition, compulsory education sports and health course standard "(hereinafter referred to as the" 2011 "), the full implementation of 18 in the third plenary session of the resolution "to strengthen the physical education, strengthen physical exercise" request is put forward, on how to improve the quality of sports teaching, how to improve the students' physical health level, etc has become the current primary and secondary school physical education teachers and school sports researchers focus (Li Youjiang, 2011). The concept of "sports" is one of the most influential theories in the school sports in the United States, it strengthens the school sports education professional, attaches great importance to the students' healthy lifestyle, such as positive triggered a new round of physical education curriculum reform, the reform results show and get international recognition. In modern times, the sports teaching the long-term scientific development needs based on the experience of the physical education reform and development of different countries, complement each other, so this article attempts to the concept of physical education and the Chinese sports teaching goals and contents of the two aspects, in order to find out the similarities and differences and the advantages and disadvantages of the two points, for the two countries to deepen and optimization of physical education teaching reform to provide the reference.

\section{OVERVIEW OF THE CONCEPT "SPORTS TEACHING" IN THE UNITED STATES}

Concept is based on the concept of physical education; adopt the teaching of primary and middle school students of biomedical, social psychology and culture method of humanities such as a set of subjects, to accomplish the aim of cultivating healthy lifestyle (hai-yong ding, Li Youjiang, 2011). It is based on sports, sports teaching sports learning principle, teaching sports learning concept as the core content, the concept of learning mainly by means of physical exercises. Sports science, the principle of physiological principle of skills related to mechanics principle in learning, etc., such as "how to control the body" and "how to control the equipment", "how to use the best way to learn a skill" and so on, is the most basic principle of sports science, is the most basic content of learning, rather than just athletic skills. Concept of sports teaching respecting the rule of the learner's cognitive development, and its deciding sports learning is associated with cognitive understanding of physical activity experience caused by the relatively permanent behavior change (Ceng Yushan, 2015). 


\section{The Concept of Physical Education and the Chinese Sports Teaching Goal}

Sports teaching goal is the result of the physical education teachers is expected to achieve and standard, is the physical education curriculum goals, curriculum goal directly affect the teaching goal to develop and design. The two countries have national standards for physical education curriculum, the two countries standards as a guidance document about school sports work, the two countries provinces or states provide basis and reference for sports teaching goal, therefore, below in the comparison of the teaching goal of sports, mainly from the Angle of the national curriculum goal compare (ministry of education, 2011).

America in 1995, 2004 and 2013 respectively introduced three versions of the National standard of primary and secondary school Physical Education curriculum, the different versions of the curriculum standard interval for nine years, the latest version of the standard is "K - 12 Physical Education Standards" (Grade - level Outcomes for k-12 Physical Education), the standard is by the association for sport and Physical Education (NASPE) council appointed course framework working group in the first two edition "into the Future - the National Physical Education Standards" (Moving into the Future: the National Standards for Physical Education) on the basis of the revision.

Primary and secondary school physical education curriculum standards in China is in the eighth elementary education curriculum reform of physical education curriculum reform, is the latest version of the course standard organization expert team to revise and in 2011 the ministry of education issued the 2011 standard, the standard is in the previous version of the full-time compulsory education (grade $1 \sim 6$ ) ordinary senior middle schools $(7 \sim 12)$ physical education (and health) curriculum standard (experiment draft) "on the basis of the compulsory education stage (grade 1-9), high school (10-12 grade) is organizing revision, temporarily not issued publication (artist jang hyuk, Tang Yan, 2015).

China in 2011, the latest edition of the (compulsory education) sports and health course standard "students can do:

- The students will master the basic knowledge of sports and health, basic skills and methods, enhance physical fitness;

- Learning to learn and exercise, the development of sports and health practice and innovation ability;

- The fun of sports and success, get into the habit of exercise;

- develop good psychological quality, cooperation and communication ability;

- To improve the maintenance of healthy consciousness, the basic form a healthy lifestyle and positive, optimistic, cheerful attitude toward life.

The United States in 2013, the latest edition of the K - 12 physical education standards "have the body quality of individuals:

- In all kinds of motor skills and movement patterns show ability;

- To the movement of the concept, principle, tactics and strategies used in the sport and performance;

- Can achieve through sports knowledge and skills, and constantly improve the level of physical activity and health level;

- Show responsible personal and social behavior, and respect for self and others in the sports activities;

- To realize sports activities for health, enjoyment, challenge, self-expression and social value.

By above knowable, standard in China and the United States there are similarities and differences in terms of goal setting.

The similarities are: (1) the target item the same number. Curriculum standards in China and the United States the target number is 5, the United States in 2013, the latest version of the K - 12 physical education standards "(hereinafter referred to as the United States [K - 12]) students should achieve the goal of prescribed standards from the first version of the article 7 whittled down to 6 to 
the latest version of the article 5, present the refined to reduce number of visible features, and the goal of China from 2001 the first edition (experimental edition) start up to now has 5, number remains the same. (2) The target concept is the same. Goals are the two countries set up take the student as the main body, embodies the "people-oriented", "take the student as the main body" the education thought and idea. In the United States "K - 12 standard target expression behaviors main body is a individual, with" literacy "and China target describe the behavior of the subject is" student ". (3) The content is concerned with the health. Target body content is designed around the student's physical and mental health, including physical, psychological and social adaptation ability.

Standard between the two countries in the aspect of goal setting differences have: (1) the action verbs describe different: the United States "K - 12 standard" words "display", "applied to" realize "action verbs, such as China in terms of target expression in words are" master ", "development", "form" and "improve", thus the action verbs in the goal setting is relative clarity verbs, have clear goal setting the action verbs in China. (2) Specific content requirements and different emphasis: China and the United States in the target design differences in how the specific content and requirements. Such as the standard 2 "to the movement of the concept, principle, tactics and strategies used in the sport and performance" this goal, for sports related knowledge of the specific expression, such as the movement of the concept, principle, tactics and strategy, etc., while Chinese expression of more general, such as "master the basic knowledge of sports and health, basic skills and methods, enhance physical fitness", for the basic knowledge of sports and health contains content relatively vague. More than the same table and differences and the history of the two countries culture and national conditions have certain relations.

\section{The Comparison of SPorts Teaching Content}

Create a "sports teaching need to be teaching content as its carrier. Concept of physical education teaching contents have a special team to research, and write the students need to master in all phases of the concept as the core of specialized teaching material, such as well, and Chen Ang people writing the sports Science and I (Science, PE and Me (grade 3, 5)), the teaching material is nearly more than 10 years of compiled on the basis of a large number of experiments, the experimental teaching materials have been used in many state schools in the United States. Is one of the prominent characteristics of the teaching material includes defining the rigorous and relevant to the health of the sports scientists verify knowledge (scientific sports concepts) and arranged in a spiral way, some important concepts and principles in different grade appeared again and again. In the teaching, teachers create the learning environment, let the students in physical activity, to understand and use this kind of scientific concepts. The teaching material content was developed by the special research team and after repeated experiments to determine the final script, has high scientific, practical and stipulation. 3 grade level, such as the teaching material of sports, about "healthy heart" unit 1 to 10 classes involved in the concept of "heart", "heart", "heart", "acuteness activity", "stopwatch" etc, each class has a corresponding teaching goal and theme, the resource of the lesson preparation, teaching process and content of the time arrangement and operation, etc.

China sports teaching content mainly referring to the national standard of "2011", each area according to the national curriculum standards for local implementation scheme of "curriculum standard", and suggests or specifies the learning content. China's "2011 standard" divide the students into four levels, learning content is divided into four aspects (sports participation, sports skills, physical health, mental health and social adaptation), different levels of four different aspects Suggestions, different levels and in study and has different learning goals. Such as sports skills, students will learn sports knowledge, master skills and methods, strengthen the consciousness of safety and prevention capacity, etc., including in the aspect of "learning sports skills and methods", learning goal 2 is the students to master the methods of a variety of sports activities, to achieve the goals, "students can preliminarily master the basic method of several sports activities". Like a ball of little basketball, soccer, badminton, table tennis or other emerging activity basic method, etc. Part of China's provinces or regions introduced related "implementation plan" sports and health course, categorize the content and the suggestion. Such as China's sports and health curriculum implementation plan of jiangsu province (trial) according to the students' physical and mental development rule and rule of motor skill learning and development of physical education teaching content can be divided into will learn to content and 
content, the limit of free content types, level ii (grade 3 to 4 ) contents of proportion for 4:4:2, and suggested that the schools in jiangsu province plans to float $5 \%$ in regulations.

By above knowable teaching content similarities between the two countries are: (1) involve health knowledge, skills, and physical fitness and so on related content; (2) the teaching contents in teaching material of the two countries are performed by team work. Differences are: (1) for "health knowledge and skills" the proportion of different contents. The core concepts about health knowledge, especially the major and system, is advantageous to the students of the core concepts of the master; But skills and fitness content relatively fragmented, this part is mainly for the core knowledge and use of the concept of service, it is not easy for students to learn the system movement skills. China sports skills in PE teaching content ratio is higher, and from the elementary school higher grades after more than with "sports" as the unit teaching, skills taught comparison system, detailed content, help students to master the skills, but health knowledge presentation teaching content trivializes situated and fragmentation characteristics. (2) the teaching content is scientific and practical. China's basic content of teaching materials by the team to write and become, on the basis of experience of different grade students should know, should be, can be the content of the lack of experimental data, so the scientific nature and the usability is low. The concept of sports teaching content is done by the team based on decades of empirical research, scientific and practicability of the content is higher. (3) the acquisition of healthy knowledge contents in a different way. China health education content usually acquisition in the interior theory, the concept of sports teaching content is usually in the sports practice. (4) teacher's choice of content different power. "2011 standard" to increase the elasticity and flexibility of PE teachers' content choice, they can be according to the national curriculum standard, with the characteristics of local and school to choose the teaching content, choice of power is larger, to arouse the teachers' creativity, suitable for experienced teachers, teachers for new teachers and non-sports professional graduate education is difficult to guarantee the content selection of science and rationality. The concept of sports teaching content and arrange scientifically rigorous, but not for physical education teachers teach content choice power of PE teachers in China. P.E. teachers for nonprofessional sports or inexperienced in terms of physical education teachers, the guarantee of power is small but it can largely teaching effect, but its limits the teachers' creativity.

\section{Conclusions}

The sports teaching target and content of the two countries have their own features and the differences and similarities, which has deep roots with history and national conditions, etc.

\subsection{Goal Setting}

Will have the same similarities with: the target number of entries, article 5; Target concept is the same, "people-oriented", the target content focused on health.

Differences are: (1) the action verbs describe different. (2) Specific content requirements and different emphasis: China and the United States requires a more specific, China's demand for healthy content relatively fuzzy, expression of more general, China for the basic knowledge of sports and health contains content relatively vague.

\subsection{Content}

Similarities are: (1) the teaching contents in teaching material of the two countries are performed by team work. (2) The teaching contents involve the two countries "health knowledge, skills, and physical fitness" and other related content. Differences are: (1) for "health knowledge and skills" the proportion of different contents. (2) The teaching content is scientific and practical. (3) The implementation of health education content in a different way. (4) Teachers for content choice of different power.

Physical education teaching quality, a long way to go, sports teachers and other researchers need to continue to learn from the past experience, study and apply the advanced achievements of different countries to explore further research.

\section{REFERENCES}

[1] Li Youjiang. American primary and middle school "sports concept" study. Journal of foreign education of primary and secondary schools, 2011, (7): 61-65.

[2] hai-yong ding, Li Youjiang. The United States "course as the center" for the concept of sports 
Teaching Objectives and Teaching Content Point of View the Concept of Sports Teaching and the Comparison of the Chinese Sports Teaching

theory and its development, the Shanghai sports institute proceedings, 2011, 35 (6) : 84-87.

[3] Ceng Yushan. The United States the new K - 12 national physical education curriculum standard analysis. Journal of Beijing sports university, 2015, (6): 109-113.

[4] The ministry of education, physical education and health curriculum standard. Beijing: Beijing normal university press, 2011:6.

[5] Artist jang hyuk, Tang Yan. The 2013 edition of "standard of k-12 physical education" characteristic and enlightenment. Journal of shenyang sports institute. 2015, (4): 115-119.

\section{AUTHOR'S BIOGRAPHY}

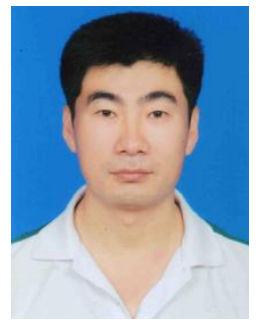

Zhang Bing (1973- ) male, Mongolian, liaoning chaoyang, master degree, associate professor, research interests: sports industry and sports engineering, sports management. 